\title{
SISTEMAS DE INFORMACIÓN EN SALUD: INTEGRANDO DATOS CLINNICOS EN DIFERENTES ESCENARIOS Y USUARIOS
}

\author{
HEALTH INFORMATION SYSTEMS: INTEGRATING CLINICAL DATA IN DIFFERENT \\ SCENARIOS AND USERS
}

\author{
Fernando Plazzotta ${ }^{1, a}$, Daniel Luna ${ }^{1, b}$, Fernán González Bernaldo de Quirós ${ }^{1, c}$ \\ Hospital Italiano de Buenos Aires. Buenos Aires, Argentina. \\ Médico, especialista en Informática Médica; ${ }^{b}$ médico, especialista en Ingeniería de Sistemas de Información; ${ }^{\mathrm{c}}$ médico, máster en Gobierno y Dirección de \\ Sistemas de Salud \\ Recibido: 22-03-15; Aprobado: 13-05-15
}

\section{RESUMEN}

Pese al avance tecnológico de las últimas décadas y a los múltiples beneficios documentados, la implementación de tecnologías de información y comunicación (TIC) en salud continúa siendo un importante desafío para las organizaciones de salud. Buscando contribuir al análisis de las múltiples variables involucradas a la hora de lograr implementaciones exitosas, aplicamos un modelo sociotécnico para analizar la experiencia de informatización de la capa clínica en el Hospital Italiano de Buenos Aires (HIBA). El marco conceptual aplicado consta de ocho dimensiones interconectadas que permiten revisar diferentes aspectos a tener en cuenta a la hora de diseñar, desarrollar, implementar, usar y evaluar la aplicación de TIC. Teniendo en cuenta la evolución de nuestro proyecto, lo dividimos en tres etapas, de forma tal que cada una de las dimensiones es analizada en cada una de las etapas. El modelo sociotécnico aplicado resultó en una adecuada herramienta de evaluación de nuestra implementación de TIC en salud, permitiéndonos el análisis de las ocho dimensiones, incluso de forma retrospectiva. Aplicando este análisis a cada una de las etapas de la evolución de nuestro proyecto institucional pudimos evidenciar que al tener en cuenta todos los aspectos en conjunto se facilitó la ejecución del mismo y nos permitió identificar aspectos por mejorar.

Palabras clave: Sistemas de información en salud; Modelos teóricos; Informática médica/organización \& administración (fuente: DeCS BIREME).

\begin{abstract}
Despite the technological advances of recent decades and the many documented benefits, the implementation of information and communication technologies (ICT) in health remains a major challenge for healthcare organizations. Looking to contribute to the analysis of multiple variables involved at the time of achieving successful implementation, we apply a sociotechnical model to analyze the experience of computerization of the clinical layer in the Italian Hospital of Buenos Aires. The conceptual framework applied consists of eight interconnected dimensions that allow the review of different aspects to consider at the time of design, development, implementation, use and evaluation of the application of ICT. Considering the evolution of our project, we divided it into three stages, so that each of the dimensions is analyzed in each of the stages. The socio-technical model applied resulted in an appropriate tool for assessing our implementation of ICT in health, allowing us the analysis of the eight dimensions, including retrospectively. Applying this analysis to each of the stages of the evolution of our institutional project we were able to show that by taking into account all aspects together, its execution was facilitated and allowed us to identify areas for improvement.
\end{abstract}

Key words: Health information systems; Models, theoretical; Medical informatics/organization \& administration (source: $\mathrm{MeSH}, \mathrm{NLM}$ ).

\section{INTRODUCCIÓN}

Las tecnologías de información y comunicación (TIC), junto con las metodologías avanzadas de procesamiento de la información han evolucionado significativamente, influyendo activamente en nuestra sociedad. En salud este impacto estaba orientado a la optimización de procesos, al incremento de productividad y la gestión financiera, en lo que conocemos como la era de los "sistemas de información hospitalarios". Sin embargo, al entender el flujo de trabajo y contexto clínico (capa clínica) como una serie de procesos complejos en los que el paciente y su interacción con el sistema de salud son el centro de la escena, estos sistemas evolucionaron hacia "sistemas de información en salud" (o sanitarios), donde la captura, intercambio, almacenamiento, acceso y gestión de información clínica adquieren un rol fundamental, con el objetivo de contribuir a la calidad y

Citar como: Plazzotta F, Luna D, González Bernaldo de Quirós F. Sistemas de información en salud: integrando datos clínicos en diferentes escenarios y usuarios. Rev Peru Med Exp Salud Publica. 2015;32(2):343-51. 
eficiencia de la atención de salud, mejorar la accesibilidad a sus servicios y el conocimiento médico (1). En este contexto, surge la informática en salud, como una disciplina que busca desarrollar y gestionar eficiente y estratégicamente estos nuevos sistemas de información sanitaria ${ }^{(2)}$.

Las TIC permiten un tratamiento sistemático de datos, información y conocimiento en el entorno sanitario y contribuyen considerablemente al progreso de las ciencias de la salud. Entre los beneficios de implementar TIC encontramos que favorecen la continuidad del cuidado gracias a que mejoran la comunicación del equipo de salud y el acceso ubicuo a la información clínica. Otros beneficios de las TIC fueron comprobados a nivel de la disminución de errores de la medicación, principalmente con la utilización de sistemas de prescripción electrónica y sistemas de soporte a la toma de decisiones ${ }^{(3)}$.

Si bien los beneficios de las TIC resultan claros y suficientes, la evidencia demuestra que la adopción de nuevos sistemas de información en la capa clínica no es una tarea fácil y las tasas de utilización no son las esperadas para el momento tecnológico que estamos viviendo, y son muy pocos los ejemplos de implementación exitosas a gran escala a nivel mundial. Esto se debe a que la informatización de la capa clínica tiene importantes barreras que pueden hacer que cualquier proyecto fracase si no son tenidas en cuenta, por ejemplo, contar con el soporte político y económico que garantice la gobernanza del proyecto, una adecuada planificación estratégica con claros objetivos a corto, mediano y largo plazo, un conjunto de normativas externas (principalmente leyes y resoluciones) e internas (procesos institucionales definidos y documentados) que ofrezcan un marco legal y regulen en estos aspectos, la definición y adopción de un conjunto de estándares que permitan integrar e interoperar datos que proceden de diferentes sistemas y, por último, un adecuado proceso de manejo del cambio organizacional en todos los niveles ${ }^{(4)}$. Es decir, la implementación de sistemas informáticos en el entorno clínico tiene condicionantes tecnológicos del sistema en sí y la infraestructura que lo soporta, pero el mayor desafío está en los condicionantes del entorno y el contexto (plan estratégico que lo enmarca, gobernanza, planificación de la formación profesional y gestión del cambio, readaptación de procesos y flujos de trabajo, reglas y leyes locales, presupuestos, comunicación, etc.) ${ }^{(5)}$.

Diversas experiencias bien documentadas a nivel mundial permiten conocer cómo han sido afrontados estos desafíos, las estrategias utilizadas y los resultados obtenidos. En su gran mayoría corresponden con países desarrollados. En el caso de América Latina y el Caribe, no existen aún implementaciones a gran escala a nivel público, destacando principalmente iniciativas de planificación estratégica a nivel gubernamental, proyectos a gran escala de aspectos puntuales y algunas del ámbito público y privado a nivel hospitalario o de pequeñas redes. Podemos destacar la estrategia gubernamental para eHealth en Brasil, Uruguay y Chile, la Red QUIPU en Perú (un proyecto para la promoción de investigación y formación de profesionales en informática biomédica y salud global), y el proyecto FEMI Salud Digital en Uruguay. En la República Argentina, varios centros cuentan con implementación de TIC, siendo la más evaluada y documentada la de la red del Hospital Italiano de Buenos Aires (HIBA) ${ }^{(6)}$.

A raíz de la importancia de aplicar un enfoque sistémico a la evaluación de implementación de las TIC, el objetivo del presente trabajo es explicar la experiencia del HIBA a través de un marco de análisis estructurado siguiendo una guía de evaluación práctica para escenarios sociotécnicos complejos ${ }^{(7)}$.

\section{MATERIALES Y MÉTODOS}

\section{ESCENARIO}

El HIBA es un hospital universitario privado sin fines de lucro que actualmente se ha transformado en una red de atención médica con dos hospitales, uno de mediana (HIBA San Justo) y otro de alta complejidad (HIBA central), 25 centros de atención primaria y ambulatoria y 250 consultorios particulares. Cuenta con 750 camas de internación, 200 de las cuales son para cuidados críticos, 41 quirófanos y más de 1000 camas de internación domiciliaria. En la red trabajan 2800 médicos, 2800 agentes del equipo de salud y 1900 personas de los sectores administrativos y de gestión. Anualmente se realizan 50000 egresos, 3 millones de consultas ambulatorias y 45000 procedimientos quirúrgicos, de los cuales el $50 \%$ son ambulatorios. Aproximadamente la mitad de los pacientes financian su atención a través de aseguradores de medicina privada y de la seguridad social. La otra mitad lo hace a través del Plan de Salud (PS) del HIBA, que funciona como un asegurador (150 000 afiliados).

Desde el año 1998 se ha implementado de manera gradual un Sistema de Información en Salud (SIS) a partir de un desarrollo in house que maneja la información sanitaria y de gestión desde la captura primaria hasta el análisis ${ }^{(8)}$. Incluye una única HCE web, modular, orientada a problemas y centrada en el paciente. Conocida con el nombre de ITÁLICA, la 
HCE permite el registro de la atención en los ámbitos ambulatorio, internación, emergencias y atención domiciliaria, con un nivel HIMSS-EMRAM 6/7 ${ }^{(9)}$. Desde el año 2007 se brinda acceso para pacientes a través de su Portal Personal de Salud (PoPeS), permitiendo acceso a tareas administrativas, resultados de estudios complementarios, información preventiva y lista de medicaciones.

\section{ESTADO BASAL DE SISTEMAS DE INFORMACIÓN}

La aplicación de las TIC en nuestra institución comienza a fines de la década del 70. Al inicio del proyecto, el HIBA tenía un sistema de información administrativo y desintegrado, compuesto por múltiples sistemas independientes, administrados por diferentes grupos no coordinados y con infraestructuras tecnológicas diversas de hardware y el software. No existían diccionarios comunes ni tablas maestras, por lo cual la interoperabilidad de estos sistemas era imposible:

- En la capa administrativa el ERP desarrollado internamente.

- Preexistían también sistemas departamentales (laboratorio, radiología, etc.) aislados.

- El plan de salud tenía su propio sistema para la gestión de los socios.

- En los servicios médicos, existían sistemas de registro de patologías (cohortes).

- En el Departamento de Docencia e Investigación con servicio de correo electrónico con dominio propio.

\section{PLAN ESTRATÉGICO}

Nos planteamos una planificación quinquenal, que podemos describir en tres etapas:

La primera etapa comenzó en 1998 que comienza con la presentación del plan estratégico y la creación de las bases que serán el sustento del proyecto y la implementación inicial de una HCE en el ámbito ambulatorio.

La segunda etapa comenzó en 2004 y es la etapa de diseminación del proyecto, generalización de los aplicativos asistenciales a todos los ámbitos y servicios e integración de fuentes de información clínicas aisladas gracias a la implementación de estándares y de los servicios terminológicos. También se sumó la posibilidad de dar acceso a pacientes a través del portal de salud.

La tercera etapa comenzó en 2010 y se caracteriza por ser una etapa de innovación y desarrollo del proyecto, con la incorporación de nuevas tecnologías, integración del usuario en el diseño de las interfaces del sistema, la orientación a la atención a distancia e incorporación de tecnologías móviles en la asistencia institucional.

\section{MARCO CONCEPTUAL}

Los sistemas complejos deben ser estudiados como múltiples componentes no lineales, emergentes y de comportamiento dinámico que interactúan entre ellos. Una metodología que refleja estas interacciones es un modelo sociotécnico de ocho dimensiones ${ }^{(7)}$, que ofrece un marco conceptual diseñado para analizar las variables sociotécnicas involucradas en el diseño, desarrollo, implementación, uso y evaluación de las TIC (Figura 1). Estas dimensiones son:

- Hardware, software e Infraestructura: esencialmente técnica. Son los equipos y aplicaciones utilizados para interactuar con el sistema.

- Contenido clínico: todos los tipos de datos e información (texto, datos numéricos, imágenes, señales biológicas, etc.), que constituyen el "lenguaje" de las aplicaciones clínicas.

- Interfaz humano-computadora: aspectos de la computadora que los usuarios puedan ver, tocar o escuchar, ya que interactúan con él.

- Gente: representa a los humanos, todos los que interactúan de alguna manera con el sistema (desde desarrolladores hasta el usuario final, incluyendo a los pacientes).

- Comunicación y procesos: entiende que el cuidado continuado se logra a través del trabajo en equipo, y para eso es necesaria la comunicación. También analiza los procesos asistenciales, de forma que los sistemas los representen correctamente.

- Características organizacionales y políticas internas: políticas, procedimientos y cultura de la organización

- Regulaciones: normativas y reglamentos externos, los cuales pueden facilitar o limitar muchos aspectos de las dimensiones anteriores.

- Medición y monitoreo: evaluación de consecuencias, tanto intencionales como no intencionales de aplicación de TIC y uso.

Proponemos un segundo eje teniendo en cuenta las tres etapas planteadas cuando hablamos de nuestra planificación quinquenal ya que, por otro lado, las ocho dimensiones no son independientes, secuenciales ni jerárquicas, sino más bien son conceptos interdependientes e interrelacionados similares a las composiciones de otros sistemas adaptativos complejos. 


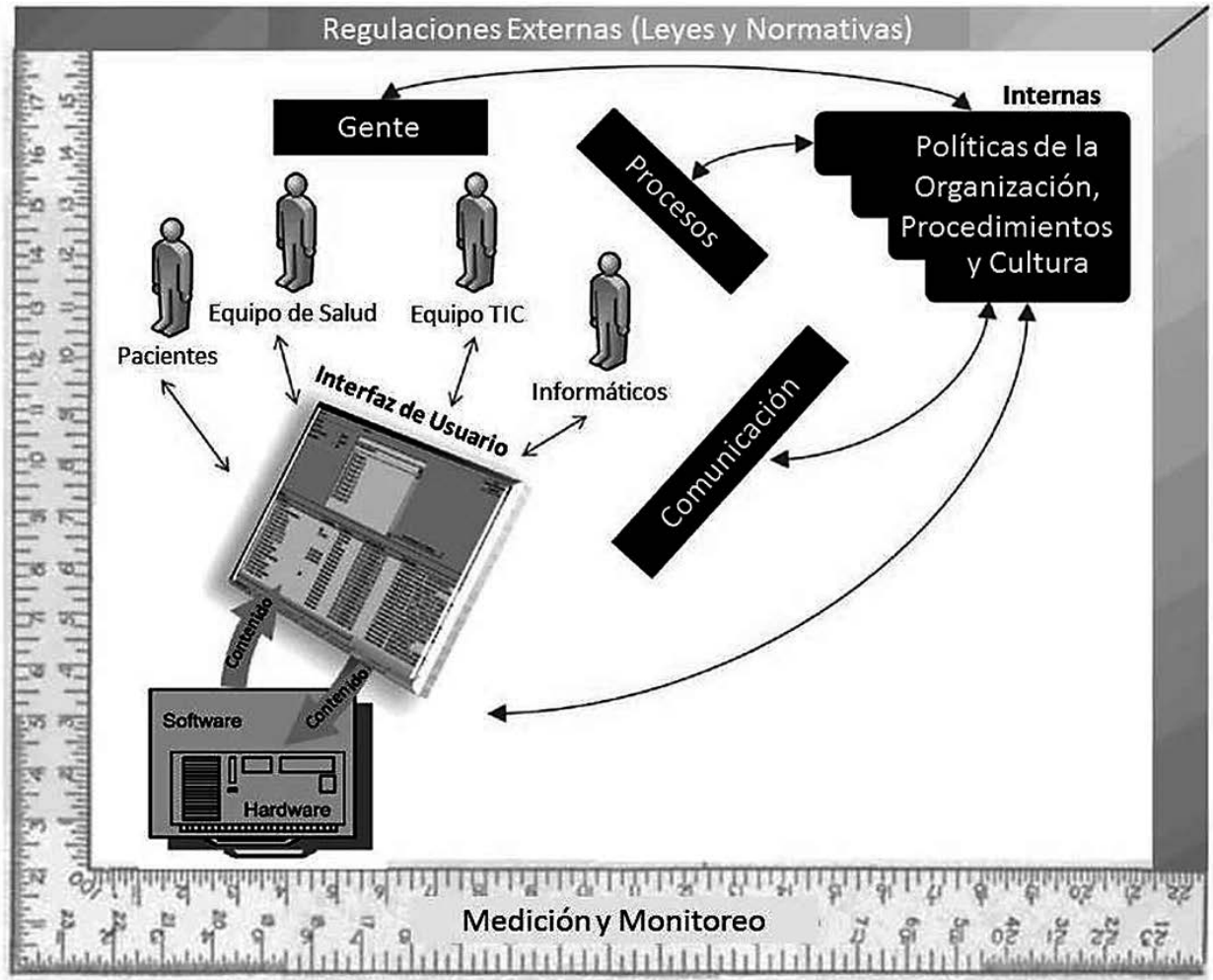

Figura 1. Muestra las relaciones complejas entre las ocho dimensiones del modelo sociotécnico.

Tomado de: Sittig DF, Singh H. A new sociotechnical model for studying health information technology in complex adaptive healthcare systems ${ }^{(7)}$

\section{RESULTADOS}

\section{HARDWARE, SOFTWARE E INFRAESTRUCTURA}

Se tomó la decisión que tanto la HCE como el resto de las aplicaciones, sistemas e interfaces sean desarrollos propios, los que nos permitió realizar las implementaciones de forma gradual, adaptativa y escalable. En la primera etapa el punto principal de esta dimensión es la integración e interoperabilidad de sistemas. Para ello, se crean grupos de trabajo de interfaces, que se ocuparon de la evaluación, estudio e implementación del estándar HL7 ${ }^{(10)}$. Al mismo tiempo, se comienza el desarrollo de diccionarios comunes para unificar el vocabulario utilizado en los mensajes. Esto permitió que la primera versión de la HCE permita la solicitud electrónica de estudios complementarios, la visualización de los estados del pedido y la recepción de resultados.

En la segunda etapa se rediseñó el Data Center, con cableado nivel 7 y una certificación Tier III. Se adquirió un core de comunicaciones de 4 unidades con 48 puertos cada una, además se realizó una actualización de todos los switches y cableados para dar soporte al incremento de tránsito de información y tipo de datos consultados. También en esta etapa se implementó una Red DICOM a gigabyte y un sistema de almacenamiento y comunicación de imágenes (PACS) dotando a la HCE con funcionalidades multimedia, incorporando visores (diagnósticos y clínicos) de imágenes radiológicas desde cualquier punto de la red, a través de la HCE ${ }^{(11,12)}$.

En esto punto del desarrollo del proyecto, la penetración del sistema en los procesos asistenciales evidenció la necesidad de lograr una alta disponibilidad de servicios. Teniendo en cuenta que la HCE cuenta con más de 5500 usuarios activos y un promedio de 15000 accesos diarios, se elaboró un plan de mejoras basado en tres aspectos: buenas prácticas y estandarización de procesos en ingeniería del software; proceso de administración y seguimiento de incidentes ITIL, y diseño de una infraestructura de alta disponibilidad (13).

Esta infraestructura de alta disponibilidad, redundante y escalable, se diseñó en tres capas: base de datos, redes y aplicaciones. En la capa de base de datos, se utilizó Oracle Real Application Clusters (RAC); en la capa de aplicaciones, HAProxy y SUN Glassfish, y en la capa de redes, se diseñó una red departamental redundante, proveyendo a los departamentos del HIBA un camino alternativo hacia el datacenter. 


\section{CONTENIDO CLÍNICO}

La correcta representación del conocimiento médico fue lográndose de forma gradual. En la primera etapa se permitió la carga de texto libre en todas las instancias de registro, con control terminológico secundario de la lista de problemas y diagnósticos de la epicrisis en internación.

En la segunda etapa se integraron todas las fuentes de información con diferentes tipos de datos, creando un único repositorio de datos clínicos. Al mismo tiempo, comenzó una estrategia de control terminológico que incluyó la utilización de SNOMED CT como terminología de referencia y la creación de un vocabulario de interfaz que permita la codificación automática de problemas, diagnósticos, procedimientos y fármacos ${ }^{(13,14) \text {. En }}$ esta etapa se implementó el estándar de documentos clínicos CDA (Clinical Document Architecture) para los informes de estudios complementarios ${ }^{(15,16)}$.

En la etapa de innovación, el servidor de terminología llegó a un punto de madurez tal que el 95\% de los textos ingresados son codificados de forma automática, permitiendo ofrecer este servicio a otras instituciones, no solo del país sino de redes asistenciales de Chile y Uruguay ${ }^{(17)}$. Al mismo tiempo, se creó un servicio de composición de fármacos que permitió representar adecuadamente la farmacopea de Argentina, Chile y Uruguay. Por último, la totalidad de los actos registrados por los profesionales en una sesión de registro fueron consolidados en documentos CDA; de esta forma se unifica la navegación de documentos clínicos para la consulta de información en la HCE ${ }^{(18)}$.

A diferencia de la dimensión anterior, el contenido clínico fue adquiriendo una importancia relativa gradual en cada una de las etapas, desde la priorización de la facilidad de uso al comienzo del proyecto, hasta la estructuración y utilidad del dato posibles en la etapa actual.

\section{INTERFAZ HUMANO-COMPUTADORA}

Al iniciar la etapa de desarrollo del software de la capa clínica, los aplicativos eran diseñados teniendo en cuenta los procesos asistenciales de cada nivel de atención (entendiendo que el repositorio de datos clínicos debía ser único): inicialmente se desarrolló la interfaz para el ambulatorio, luego para la internación, luego la de guardia y por último, internación domiciliaria ${ }^{(19)}$.

En la segunda etapa se aplicaron técnicas de prototipado para el diseño de la primera versión del PoPeS (20). Gradualmente se fueron sumando funcionalidades a la HCE, permitiendo la prescripción de fármacos y la evolución diaria en internación. Al finalizar esta etapa se planteó la necesidad de integrar las diferentes interfaces de la HCE en un único portal asistencial, pero preservando las funcionalidades según cada nivel.

La tercer etapa se decidió crear un equipo especializado en diseño centrado en el usuario y un laboratorio de usabilidad que rediseñó íntegramente la HCE con una única interfaz para todos los ámbitos asistenciales $y$, gradualmente, se aplicaron técnicas de diseño de interacciones para la prescripción electrónica y otras secciones críticas $(21,22,23)$.

\section{GENTE (PEOPLEWARE)}

Tomando como premisa que el componente organizacional y la gestión del cambio representan un $80 \%$ del éxito del proyecto ${ }^{(24)}$, en la etapa inicial se buscó la participación de profesionales de toda la institución mediante la creación de un comité de registro médico. Al comienzo del proyecto se dio mayor importancia a la atención primaria, y luego de forma gradual fueron incorporándose las diferentes especialidades. Todos los grupos de sistemas se integraron en un Departamento de Informática en Salud, y se creó una instancia de especialización en esta disciplina para profesionales médicos a través de una Residencia de Informática Médica, encargados de la capacitación y el soporte de los procesos asistenciales ${ }^{(23,24)}$.

En la segunda etapa se formalizaron los canales de capacitación de los usuarios, con nuevas estrategias (cursos online con entornos de práctica de la HCE). Una estrategia de incentivo para el uso de la HCE en esta etapa fue la creación de ingresos estructurados para las diferentes especialidades, eliminando registros secundarios para la investigación.

En esta dimensión los puntos fuertes estuvieron al principio del proyecto y en la etapa actual. EI DIS superó las 160 personas, se implementaron cursos de gestión de proyectos y mentoring para líderes, se creó una estructura matricial, transversal a las áreas (y profesiones) del departamento a través de equipos de trabajo multidisciplinarios. En concordancia con otros dominios (principalmente interfaz humanocomputadora y comunicación), esta etapa se caracterizó por un incremento en la participación de los usuarios (profesionales de la salud y pacientes) en el diseño del sistema, así como diferentes estrategias de comunicación sobre cambios y futuras funcionalidades.

\section{COMUNICACIÓN Y PROCESOS}

En las etapas iniciales se buscó crear nuevos canales de comunicación para dar soporte a un proceso de 
transformación bidireccional (sistema y organización) como consecuencia de la implementación de un SIS. Se concibió una intranet institucional como portal de acceso a todos los sistemas, con noticias institucionales y de interés general, una casilla de correo institucional y la posibilidad de realizar sugerencias en todos los aplicativos para lograr una adecuada retroalimentación por parte de los usuarios.

En la segunda etapa se logró que el área encargada del análisis de procesos y generación de normas institucionales pase a depender del departamento de informática en salud, para acompañar el relevamiento de sistemas con el de procesos.

Al tener prácticamente todos los procesos asistenciales representados en el sistema de información, no fue necesario tener definidos los circuitos alternativos para garantizar el cuidado de la salud cuando el sistema no funciona. En la última etapa se hizo foco en lograr un procedimiento de contingencia para operar de forma coordinada en los cortes programados y enfrentar la incertidumbre en los no programados ${ }^{(27)}$.

\section{POLÍTICAS ORGANIZACIONALES INTERNAS, PROCEDIMIENTOS Y CULTURA}

El HIBA inició hace 18 años un ambicioso plan estratégico que incluyo el rediseño de su red de provisión de servicios. Este proyecto abarcó estrategias de desarrollo de competencias profesionales en la búsqueda de la excelencia, una política de continuidad y coordinación de cuidados en la red, la descentralización y rediseño de sus estructuras físicas, una inversión significativa en equipamiento médico avanzado y de infraestructura.

Nuestro SIS es una herramienta que logra colaborar con el cumplimento de los objetivos de la organización de manera eficiente y efectiva, contribuyendo con la mejora de procesos asistenciales, de educación e investigación, de administración y gestión de la red. Siguiendo este enfoque estratégico, consideramos que el proyecto requería un Departamento Profesional (DIS), bajo la dirección de un médico especialista en sistemas de información, con profesionales de diferentes ámbitos y disciplinas, que conforman un equipo de trabajo transdisciplinario.

Todos los sistemas que se implementan llevan una revisión de los procesos asistenciales que impactan y la definición de normas y procedimientos para establecer y documentarlos. Esta es la justificación por la cual el área que define las normas y procedimientos de la institución pasó a formar parte del DIS, participando directamente en el circuito de ingeniería del software.

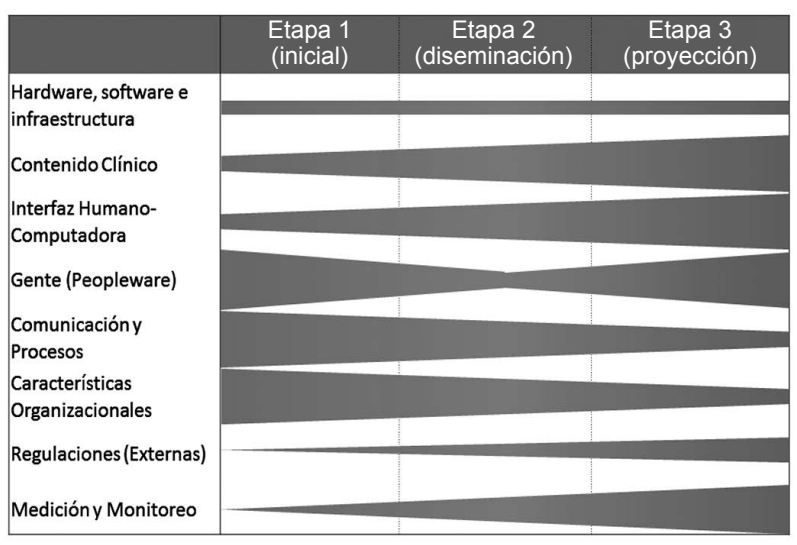

Figura 2. Importancia relativa observada en cada etapa para cada una de las dimensiones

\section{REGULACIONES Y PRESIONES EXTERNAS}

Sin una ley que defina específicamente la validez de la HCE en nuestro país, fue necesario documentar todos los procedimientos de backup de información y registros de acceso a las bases de datos. En cuanto a la prescripción farmacológica, solo se pudieron reemplazar las recetas en papel para la farmacia del hospital, ya que la legislación local requiere la escritura manuscrita del genérico indicado.

Gradualmente, el marco legal nacional fue incorporando normativas, que si bien no aplicaban directamente a la $\mathrm{HCE}$, indirectamente abarcaban alguno de sus aspectos (firma digital, protección de datos personales, derechos del paciente, etc.). Estas leyes dotaron de validez a los documentos electrónicos, permitiéndonos iniciar una estrategia de digitalización de historias clínicas antiguas, con un importante ahorro de espacio físico de almacenamiento. Estos documentos digitalizados son accesibles desde la HCE.

En concordancia con normativas vigentes, el PoPeS permite que los pacientes accedan a información sobre los estudios complementarios que tienen indicados, la preparación necesaria, el consentimiento informado y el informe con los resultados ${ }^{(28,29)}$.

\section{MONITOREO Y MEDICIÓN}

Un programa efectivo e medición y monitoreo del sistema debe tener en cuenta cuatro aspectos relacionados con las características y funcionalidades de las TIC: la disponibilidad, uso, efectividad asistencial y su impacto ${ }^{(30)}$.

La monitorización de incidentes y su seguimiento está a cargo de la mesa de ayuda (donde se registran las 
solicitudes de soporte, incluyendo cortes y caídas del sistema). Al tener registrado los incidentes denunciados, la mesa de ayuda fue capaz de generar indicadores de disponibilidad de servicios como así también de causas que provocaron la no disponibilidad de servicio.

En cuanto al uso, efectividad e impacto del sistema de información. El DIS, en sintonía con la misión institucional, prioriza la investigación y la evaluación continua de las herramientas que desarrolla, así como las posibles innovaciones para su mejora, para ello se creó el Centro de Investigación y Educación en Tecnología e Informática para la Salud (CIETIS), que lleva adelante el portfolio de investigación del departamento. En los últimos 15 años la producción científica del grupo profesional encargado del proyecto supera las 70 publicaciones en revistas y 100 presentaciones en congresos, en ambos casos nacionales, regionales y mundiales ${ }^{(6)}$.

\section{CONCLUSIÓN Y LECCIONES APRENDIDAS}

Estas ocho dimensiones propuestas deben tomarse como un conjunto. En muchos casos se toman decisiones o analizan casos teniendo en cuenta exclusivamente la problemática del software, y esto representa solo una parte de una de las dimensiones.

El modelo sociotécnico aplicado resulta en una adecuada herramienta de evaluación de intervenciones e implementaciones de TIC en salud, que permite el análisis de las ocho dimensiones, incluso de forma retrospectiva. De esta forma pudimos aplicarlo a cada una de las etapas de la evolución de nuestro proyecto institucional.

Aplicando este análisis a nuestra experiencia, pudimos evidenciar que al tomar en consideración todas las dimensiones, se hizo más fácil y factible la implementación del proyecto. Por otro lado, nos permitió identificar algunos aspectos en los cuales debíamos poner un foco prioritario, por el impacto que tenía en múltiples dimensiones (por ejemplo, monitorización de disponibilidad).

Nuestro enfoque tiene un importante foco en la creación y mantenimiento de equipos de trabajo competentes y profesionales. Consideramos que esta experiencia nos generó un importante aprendizaje, el cual resumimos a través del siguiente decálogo de lecciones aprendidas:

- Debe evitarse el falso debate entre gestión y asistencia sanitaria. Si se busca una gestión más eficiente, se debe priorizar una misión y proyectos comunes, y la visión de la asistencia profesional de cómo construirla en el día a día.

- Las nuevas demandas de manejo de enfermedades crónicas y la mejora de la relación calidad/costos requieren indispensablemente coordinación y continuidad de cuidados entre todos las especialidades y niveles de complejidad. La continuidad e integridad de la información de la HCE son críticos para este objetivo.

- El modelo de gobierno de los proyectos informáticos, para ser sustentable, debe basarse en la misión y valores de la organización, más que en el apoyo político de personas que circunstancialmente tienen la responsabilidad de conducción. Son proyectos a largo plazo, durante los cuales las personas responsables pueden cambiar, en especial en el sector público.

- Un grupo de informáticos con un grupo de médicos y enfermeros no forman un equipo de informática en salud. Se necesitan personas que hagan de "puente" entre las dos disciplinas y culturas y que tengan formación en Informática en Salud (partiendo de distintas disciplinas).

- El software no debe ser un instrumento de cambio cultural o del proceso. Los cambios deben iniciarse antes de intentar implementar las aplicaciones. Los procesos no deben adaptarse al sistema, sino que deben rediseñarse en conjunto y el sistema garantizar su cumplimiento.

- El equipo de salud suele tener poca resistencia al cambio, excepto cuando el cambio está fuera de su control o busca modificar las condiciones imperantes (lo cual ocurre frecuentemente). Cuando las aplicaciones no consideran las necesidades asistenciales, el equipo de salud tiene múltiples formas de resistencia, siendo la más común el uso incorrecto de los sistemas y en consecuencia, la mala calidad de la información y los riesgos en la seguridad de los pacientes.

- La codificación y control semántico no deben interrumpir el proceso de registro ni alterar la atención. Para tener un sistema de información clínica de alta calidad, la captura primaria del dato debe ser lo más expresiva posible (texto libre).

- El $80 \%$ del éxito de un proyecto informático está en el peopleware, el cual está muy condicionado por el contexto local y su cultura.

- Hay una gran diferencia entre guardar datos en la computadora y crear conocimiento para ser capaz de modificar la ecuación calidad/costos, y que esto modifique los indicadores sanitarios.

- En las organizaciones sanitarias, el "producto" lo construye un profesional según su mejor saber 
y entender. Este se produce bajo la observación directa del cliente (paciente). Estas condiciones hacen que la información y el sistema que la maneja, estén en el core business de la organización.
- Se puede tercerizar la adquisición de la herramienta (software) pero no el conocimiento sobre la disciplina ni el diseño del proyecto o la evaluación del impacto del mismo.

\section{REFERENCIAS BIBLIOGRÁFICAS}

1. Haux R. Health information systems - past, present, future. Int J Med Inform. 2006 Mar-Apr;75(3-4):268-81.

2. Hersh WR. Medical informatics: improving health care through information. JAMA. 2002 Oct 2330;288(16):1955-8.

3. Bright TJ, Wong A, Dhurjati R, Bristow E, Bastian L, Coeytaux RR, et al. Effect of clinical decision-support systems: a systematic review. Ann Intern Med. 2012 Jul 3;157(1):2943. doi: 10.7326/0003-4819-157-1201207030-00450.

4. Ajami S, Bagheri-Tadi T. Barriers for Adopting Electronic Health Records (EHRs) by Physicians. Acta Inform Med. 2013;21(2):129-34. doi: 10.5455/aim.2013.21.129-134.

5. Ash JS, Bates DW. Factors and forces affecting EHR system adoption: report of a 2004 ACMI discussion. J Am Med Inform Assoc. 2005 JanFeb;12(1):8-12.

6. González Bernaldo de Quiros F, Luna DR, Baum AJ, Plazzotta F, Otero C, Benitez SE. Incorporación de tecnologías de la información y de las comunicaciones en el Hospital Italiano de Buenos Aires [Internet]. 2014 [citado el 21 de marzo de 2015]. Available from: http://www.cepal.org/ es/publicaciones/3959-incorporacionde-tecnologias-de-la-informacion-y-delas-comunicaciones-en-el

7. Sittig DF, Singh H. A new sociotechnical model for studying health information technology in complex adaptive healthcare systems. Qual Saf Health Care. 2010 Oct;19 Suppl 3:i68-74. doi: 10.1136/ qshc.2010.042085.

8. Luna D, Otero P, García Martí S, López Osornio A, de los Rios E, Pedernera $\mathrm{F}$, et al. Implementación de una Historia Clínica Electrónica Ambulatoria: "Proyecto ITALICA" [Internet]. Paper presented at the 6to Simposio de Informática en Salud 32 JAIIO, Buenos Aires, Argentina.
2-5 Septiembre 2003 [citado el 21 de enero de 2015]. Disponible en: http://www.researchgate. net/publication/257935456 Implementacin_de_una_Historia_ Clnica_Electrnica_Ambulatoria_El_ Proyecto_Itlica

9. Healthcare Information and Management Systems Society (HIMSS). Electronic Medical Record Adoption Model (EMRAM) ${ }^{\mathrm{SM}}$ [Internet]. Chicago: HIMSS; c2015 [citado el 21 de enero de 2015]. Available from: http://www.himssanalytics.org/ emram/emram.aspx

10. Gomez A, Kaminker D, Campos F, Sarandria G, Calvo D, Luna D, et al. Implementación de un sistema de mensajeria electrónica (HL7) para la integración de un sistema multiplataforma. Paper presented at the 4to Simposio de Informática en Salud - 30 JAIIO, Buenos Aires, Argentina. Sociedad Argentina de Informática e Investigación Operativa (SADIO); 2001. Disponible en: http://www.researchgate. net/publication/260779643 Implementacin_de_un_sistema_ de_mensajera_electrnica_(HL7)_ para_la_integracin_de_un_sistema_ multiplataforma

11. Plazzotta F, Kaminker D, Campos F, Cancio A, Luna D, García R, et al. Imágenes más allá del PACS: el Proyecto Historia Clínica Multimedia [Internet]. II Congreso Argentino de Informática Médica - III Congreso Latinoamericano de Informática Médica - II Simposio Argentino de Informática en Enfermería. Buenos Aires, Argentina. 29, 30 y 31 de octubre y $1^{\circ}$ de noviembre de 2008 [citado el 21 de enero de 2015]. Disponible en: http://www.hospitalitaliano.org.ar/ archivos/servicios_attachs/4826.pdf

12. Soriano E, Plazzotta F, Campos F, Kaminker D, Cancio A, Aguilera Díaz J, et al. Integration of healthcare information: from enterprise PACS to patient centered multimedia health record. Stud Health Technol Inform. 2010;160(Pt 1):126-30.

13. Cancio AH, Merli M, Pintos L, Campos F, Luna DR. Plan de mejoras en TI para optimizar la disponibilidad de servicio de la Historia Clínica Electrónica [Internet]. Conferencia Latinoamericana de Informática Médica. Montevideo, Uruguay. Sociedad Uruguaya de Estandarización, Intercambio e Integración de Datos e Información de Servicios de Salud (SUEIIDIS) y Sociedad Uruguaya de Informática en Salud (SUIR). 16 y 17 de octubre de 2014 [citado el 21 de enero de 2015]. Disponible en: http:// www.hospitalitaliano.org.ar/infomed/ index.php? contenido=ver_curso. php\&id_curso $=16949 \#$.VWxv5M9 NBd

14. Osornio AL, Luna D, Gambarte ML, Gomez A, Reynoso G, de Quirós FGB. Creation of a local interface terminology to SNOMED CT. Stud Health Technol Inform. 2007;129(Pt 1):765-9.

15. Gambarte ML, Osornio AL, Martinez M, Reynoso G, Luna D, de Quiros FGB. A practical approach to advanced terminology services in health information systems. Stud Health Technol Inform. 2007;129(Pt 1):621-5.

16. Campos F, Kaminker D, Plazzotta F, Cancio A, Soriano E, Luna DR, et al. "Show me Your CDA": structured report editor for the hiba multimedia EHR. Interfaces. 2009;(April):1-14.

17. Luna D, Lopez G, Otero C, Mauro A, Casanelli CT, de Quirós FGB. Implementation of interinstitutional and transnational remote terminology services. AMIA Annu Symp Proc. 2010 Nov 13;2010:482-6.

18. Campos F, Plazzotta F, Luna D, Baum A, de Quirós FGB. Developing and implementing an interoperable document-based electronic health record. Stud Health Technol Inform. 2013;192:1169.

19. Aguilera Díaz J, Arias AE, Budalich 
CM, Benítez SE, López G, Borbolla D, et al. Development and implementation of an integrated EHR for Homecare Service: a South American experience. Stud Health Technol Inform. 2010;160(Pt 1):43-7.

20. Baum A, Mauro A, Borbolla D, Luna D, González F, de Quirós BD. Evaluating designs against users' requirements in Latin America : an experience of human-centred methods, techniques and tools on a low budget. In: Kuhn KA, Warren JR, Leong TY (ed). Medinfo 2007. Proceeding of the 12th World Congress on Health (Medical) Informatics. Netherland: IOS Press; 2007.

21. Rizzato D, Mayan J, García MJ, Smith MI, Benitez SE, Baum A, et al. Desafíos en la prescripción segura de medicamentos: diseño e implementación de una herramienta electrónica de reconciliación en el Hospital Italiano de Buenos Aires [Internet]. Conferencia Latinoamericana de Informática Médica. Montevideo, Uruguay. Sociedad Uruguaya de Estandarización, Intercambio e Integración de Datos e Información de Servicios de Salud (SUEIIDIS) y Sociedad Uruguaya de Informática en Salud (SUIR). 16 y 17 de octubre de 2014 [citado el 21 de enero de 2015]. Disponible en: http:// www.hospitalitaliano.org.ar/infomed/ index.php?contenido=ver_curso. php\&id_curso $=16949 \#$.VWxv5M9 NBd

22. Luna D, Stanziola E, Otero C, Quispe M, Belloso W, Risk M.
Diseño participativo de alertas para la Interacción Droga - Droga en una Historia Clínica Electrónica [Internet]. Conferencia Latinoamericana de Informática Médica. Montevideo, Uruguay. Sociedad Uruguaya de Estandarización, Intercambio e Integración de Datos e Información de Servicios de Salud (SUEIIDIS) y Sociedad Uruguaya de Informática en Salud (SUIR). 16 y 17 de octubre de 2014 [citado el 21 de enero de 2015]. Disponible en: http://www. hospitalitaliano.org.ar/infomed/index. php?contenido=ver_curso.php\&id curso $=16949 \#$.VWxv5M9_NBd

23. Taliercio V, Schachner B, Borbolla D, Luna D, Villalba E, Quiros F. The expectations of nurses about the implementation of a Barcoded Medication Administration System: a qualitative study. Stud Health Technol Inform. 2014;205:191-5.

24. Lorenzi NM, Riley RT. Managing Change: an overview. J Am Med Inform Assoc. 2000 Mar-Apr;7(2):116-24.

25. Baum AJ, Plazzotta F, Canosa D, Borbolla DA, Otero PD, Daniel R. Especialistas en Informática Médica : 10 años de experiencia de un porgrama de residencias médicas en Sudamérica. Infolac (2011-05). Guadalajara; 2006.

26. Gonzalez Bernaldo de Quiros F, Luna D, Otero P, Baum A, Borbolla D. Spreading knowledge in medical informatics: the contribution of the hospital Italiano de Buenos Aires. Yearb Med Inform. 2009;147-52.
27. Fernandez M, Gómez A, Santojani A, Cancio AH, Luna DR, Benitez SE. Electronic Health Record System Contingency Plan Coordination: A Strategy for Continuity of Care Considering Users's Needs.

28. Borbolla D, Del Fiol G, Taliercio V, Otero C, Campos F, Martinez M, et al. Integrating personalized health information from MedlinePlus in a patient portal. Stud Health Technol Inform. 2014;205:348-52.

29. Martinez M, Baum A, Saldaño AMG, Gomez A, Luna D, De Quirós FGB. Predictive variables of the use of personal health record: The Hospital Italiano de Buenos Aires study. Stud Health Technol Inform. 2013;192:1171.

30. Leonard KJ, Sittig DF. Improving information technology adoption and implementation through the identification of appropriate benefits: creating IMPROVE-IT. J Med Internet Res. 2007 May 4;9(2):e9.

Correspondencia: Fernando Plazzotta.

Dirección: Araoz 2754 3. ${ }^{\circ}$ G. Ciudad Autónoma de Buenos Aires (1425), Argentina.

Teléfono: (54-11) 3396-0986.

Correo electrónico: fernando.plazzotta@

hospitalitaliano.org.ar 